\title{
Effect of intermediate care on mortality following emergency abdominal surgery. The InCare trial: study protocol, rationale and feasibility of a randomised multicentre trial
}

Morten Vester-Andersen ${ }^{1 *}$, Tina Waldau', Jørn Wetterslev², Morten Hylander Møller ${ }^{3}$, Jacob Rosenberg ${ }^{4}$, Lars Nannestad Jørgensen ${ }^{5}$, Inger Gillesberg ${ }^{1}$, Henrik Loft Jakobsen ${ }^{4}$, Egon Godthåb Hansen ${ }^{1}$, Lone Musaeus Poulsen ${ }^{6}$, Jan Skovdal ${ }^{7}$, Ellen Kristine Søgaard ${ }^{6}$, Morten Bestle ${ }^{8}$, Jesper Vilandt ${ }^{9}$, Iben Rosenberg ${ }^{8}$, Rasmus Ehrenfried Berthelsen ${ }^{8}$, Jens Pedersen ${ }^{10}$, Mogens Rørbæk Madsen ${ }^{11}$, Thomas Feurstein ${ }^{12}$, Malene Just Busse ${ }^{13}$, Johnny D H Andersen ${ }^{12}$, Christian Maschmann ${ }^{14}$, Morten Rasmussen ${ }^{5}$, Christian Jessen ${ }^{15}$, Lasse Bugge $^{16}$, Helle Ørding ${ }^{15}$ and Ann Merete Møller ${ }^{1}$

\begin{abstract}
Background: Emergency abdominal surgery carries a 15\% to 20\% short-term mortality rate. Postoperative medical complications are strongly associated with increased mortality. Recent research suggests that timely recognition and effective management of complications may reduce mortality. The aim of the present trial is to evaluate the effect of postoperative intermediate care following emergency major abdominal surgery in high-risk patients.

Methods and design: The InCare trial is a randomised, parallel-group, non-blinded clinical trial with 1:1 allocation. Patients undergoing emergency laparotomy or laparoscopic surgery with a perioperative Acute Physiology and Chronic Health Evaluation II score of 10 or above, who are ready to be transferred to the surgical ward within $24 \mathrm{~h}$ of surgery are allocated to either intermediate care for $48 \mathrm{~h}$, or surgical ward care. The primary outcome measure is all-cause 30-day mortality. We aim to enrol 400 patients in seven Danish hospitals. The sample size allows us to detect or refute a $34 \%$ relative risk reduction of mortality with $80 \%$ power.

Discussion: This trial evaluates the benefits and possible harm of intermediate care. The results may potentially influence the survival of many high-risk surgical patients. As a pioneer trial in the area, it will provide important data on the feasibility of future large-scale randomised clinical trials evaluating different levels of postoperative care.
\end{abstract}

Trial registration: Clinicaltrials.gov identifier: NCT01209663

Keywords: Emergency, Surgery, APACHE II score, Intermediate care, High-dependency unit, Postoperative care, Clinical trial, Randomised, Mortality, Length of stay

\footnotetext{
* Correspondence: drvester@dadlnet.dk

'Department of Anaesthesiology and Intensive Care Medicine, Herlev Hospital, Copenhagen University, Herlev Ringvej 75, DK-2730 Herlev, Denmark

Full list of author information is available at the end of the article
} 


\section{Background}

Worldwide an estimated 234 million surgical procedures are performed every year [1]. Overall, non-cardiac surgery carries low postoperative mortality rates of $1.4 \%$ to $1.9 \%$ [2-4]. However, this conceals the fact that a subgroup has a high risk of postoperative death. This highrisk group comprises patients often of advanced age with significant co-existent disease undergoing complex emergency surgery [4]. Emergency abdominal surgery carries a $15 \%$ to $20 \%$ short-term mortality rate [5-8], with cardiopulmonary complications and sepsis as the most frequent causes of death [9]. Postoperative medical complications are more important determinants of postoperative death than preoperative demographic characteristics, and intraoperative adverse events $[8,10]$. Recent research suggests that timely recognition, and effective management, of postoperative complications may reduce mortality [11]. Thus, early routine postoperative admission of high-risk non-cardiac surgical patients to intensive or intermediate care units could prove important. However, many countries have limited access to these facilities $[4,12]$. The majority of high-risk non-cardiac surgical patients are consequently treated on standard surgical wards with restricted resources for monitoring, and advanced treatment methods [4,13]. Intermediate care (IC) could be an appropriate level of care for stable postoperative patients with an a priori high risk of complications and death. IC is generally defined as a level of care intermediate between that provided by a general ward and an intensive care unit. An IC unit monitors and supports patients with, or likely to develop, acute (or acute on chronic) single organ failure [14-16]. The effect of postoperative IC compared to ward care on high-risk surgical patients' outcome is only sparsely evaluated, and never in a randomised clinical trial [17-23] (Additional file 1: Search string).

The aim of the InCare trial is to evaluate the effect of postoperative IC following emergency major abdominal surgery in high-risk patients identified by an Acute Physiology and Chronic Health Evaluation (APACHE) II score of 10 or above. The APACHE II score is a well-established classification system of the severity of diseases used in intensive care units worldwide [24], which can be used on emergency major abdominal surgical patients as well [5,25-31]. Emergency major abdominal surgical patients with an APACHE II score of 10 to 11 and above have a $28 \%$ to $45 \%$ mortality rate compared to patients with an APACHE II score below 9 and 10 who evidence a mortality rate of $0 \%$ to $7 \%$ [27-31]. We hypothesise that postoperative IC will lead to a reduction in postoperative mortality by avoidance of, or timely recognition and effective management of, postoperative complications. Furthermore, we hypothesise that early admission to postoperative IC may reduce later admission to intensive care.

\section{Methods and design}

\section{Trial design}

The InCare trial is an ongoing multicentre, randomised, parallel-group, non-blinded clinical trial with 1:1 allocation. Emergency abdominal surgical patients are postoperatively allocated to either: (1) IC for $48 \mathrm{~h}$; or (2) surgical ward care (standard treatment). The trial was initiated in October 2010, and we aim to enrol 400 patients.

\section{Setting}

In Denmark all emergency surgical patients are treated in tax-financed public-healthcare centres. The total intensive and intermediate care availability in Denmark is 8.3 beds per 100,000 citizens in 2010, with seven intensive care beds per 100,000 citizens and 1.3 intermediate care beds per 100,000 citizens (unpublished data from The Danish Regions central office). In comparison the intensive care availability in the United States was 20.0 per 100,000 citizens, and in the United Kingdom it was 3.5 per 100,000 citizens in 2005 [32]. Currently seven Danish tertiary referral university affiliated publichealthcare centres are participating in the trial.

\section{Inclusion criteria}

Patients are eligible for inclusion if: (1) they have undergone emergency gastrointestinal laparotomy or laparoscopic surgery; (2) they are ready to be transferred to the ward after a postoperative stay in a post-anaesthesia care unit or an intermediate/intensive care unit for $<24 \mathrm{~h}$; and (3) they have a perioperative APACHE II score of $\geq 10$. We define emergency surgery as indicated surgery, which should be undertaken within $24 \mathrm{~h}$.

Because of slower enrolment rate than anticipated, the steering committee have decided to include patients with Apache II scores of 10 and 11 although the original inclusion criteria was an Apache II score $\geq 12$. This was initiated on 23 May 2012 after 192 enrolled patients. Patients with Apache II scores of 10 and 11 also have a high 30-day mortality and the potential to benefit from the IC intervention [27-31].

\section{Exclusion criteria}

Exclusion criteria are as follows: (1) appendectomy; (2) laparoscopic cholecystectomy; (3) negative diagnostic laparoscopy; (4) intensive care not indicated (patients on palliative care, or with irreversible organ failure); (5) previous participation in the trial; (6) age <18 years; (7) trauma; and (8) no IC bed available.

\section{Recruitment, screening and enrolment}

We recruit patients postoperatively in the post-anaesthesia care unit and intensive/intermediate care unit. All adult patients who have undergone the relevant surgical procedures have a venous and arterial blood gas sample taken 
upon arrival from the operating theatre. Uniformity of trial site standard postoperative vital-sign monitoring is ensured, enabling calculation of the APACHE II score, when patients are ready to be transferred to the surgical ward in accordance with the trial site's discharge criteria - local adjusted versions of the Danish national recommendations [33] (Additional file 2: National discharge recommendations). At this point of time the attending anaesthetist screens for trial eligibility using a standard screening form with flowcharts (Figure 1). If none of the exclusion criteria 1 to 7 apply, the APACHE II score is calculated from the worst parameter values measured $12 \mathrm{~h}$ preoperatively, peroperatively, and up to $12 \mathrm{~h}$ postoperatively. If there is missing data for the APACHE II score calculation, when the patient is transferable to the ward, current values are used. GCS score, blood pressure and heart rate recorded during anaesthesia are not included in the APACHE II score calculation. The APACHE II score is calculated electronically on the InCare trial website [34]. If the patient has an APACHE II score of $\geq 10$ the anaesthetist checks that an IC bed can be established at the intermediate care, intensive care or postanaesthesia care unit. If an IC bed is not available, the patient is registered as 'not included due to lack of IC bed' and transferred to the ward. If an IC bed is available, written consent is obtained from the patient or a legal representative, if the patient is incapable. If it is not possible to contact a legal representative within $2 \mathrm{~h}$, the patient is included in the trial, and written consent is obtained as soon as possible.

\section{Randomisation}

The patients are randomised using a telephone-based central interactive-voice-response system managed by the Copenhagen Trial Unit (CTU). The randomisation system is accessible around the clock. It ensures immediate computer-based allocation, and adequate allocation concealment. A computer-generated block randomisation, with the investigators unknown and varying block size is used. Stratification variables are: trial site; APACHE II score (10 to 14 or $\geq 15$ ); and perforated viscera (yes versus no).

\section{Interventions \\ Intermediate care}

IC is initiated as soon as the patient is allocated, and continues for at least $48 \mathrm{~h}$. The IC bed is situated at an intermediate care, an intensive care or a postanaesthesia care unit, which can provide the outlined intervention (Table 1). IC is defined as a minimal monitoring level and maximal treatment level. The minimal monitoring level is applied when the patient's vital signs are stable. If the patient deteriorates, the level of monitoring and treatment is increased as indicated. When the maximal level of treatment defined in the IC is exceeded, that is when there is a need for invasive ventilation, emergency dialysis, parallel sympathomimetic drug infusion or invasive arterial-blood pressure monitoring, the patient is categorised as an intensive care patient. However, the patient remains in the trial for observation of the outcome measures. Surgeons and intensivists make compulsory protocol-based rounds on a daily basis using a standard form (Additional file 3: Surgeon - protocolbased round and Additional file 4: Intensivist - protocolbased round). All medical treatment and investigations are made on medical indication alone, not determined by the trial-protocol. Forty-eight hours after randomisation, or at morning handover if the patient is included at

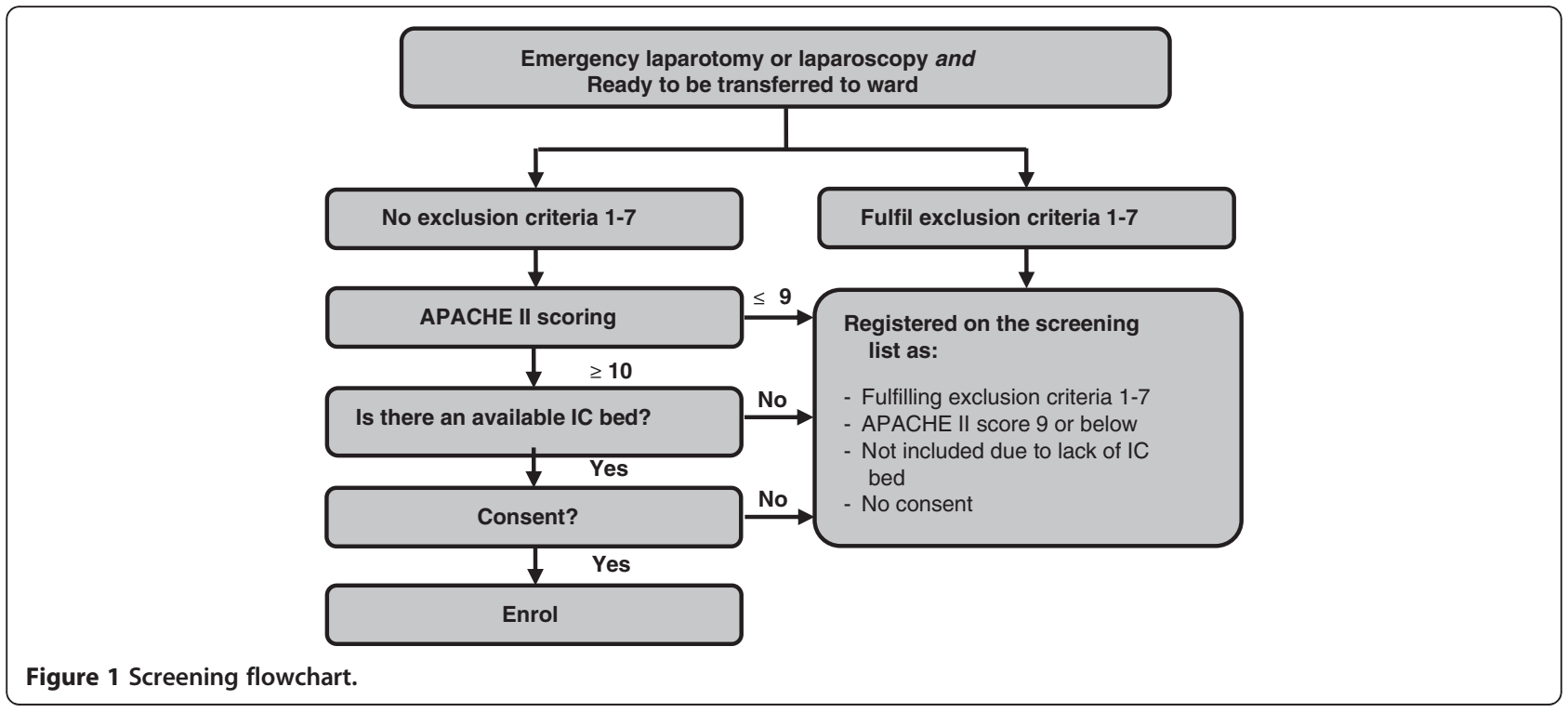


Table 1 Definition of intermediate care

\begin{tabular}{|c|c|c|c|}
\hline Observation & Minimal monitoring level ${ }^{\mathrm{a}}$ & Treatments goals $^{\mathbf{b}}$ & Comments \\
\hline Level of consciousness & Every $8 \mathrm{~h}$ & GCS: 15 & \\
\hline Respiratory rate & Every second hour & RR: 10 to 20 & $\begin{array}{l}\text { If the patient has stable vital signs the } \\
\text { RR is not measured during nights }\end{array}$ \\
\hline Oxygenation & Continuous pulse oximetry & $\mathrm{SpO}_{2} \geq 94 \%$ & $\begin{array}{l}\text { Continuous pulse oximetry when the } \\
\text { patient is supine or sitting in a chair. } \\
\text { Discontinued during mobilisation }\end{array}$ \\
\hline Blood pressure & Every second hour & MAP: 65 to $110 \mathrm{mmHg}$ & $\begin{array}{l}\text { If the patient has stable vital signs the } \\
\text { MAP is not measured during nights }\end{array}$ \\
\hline \multirow[t]{2}{*}{ Heart rate } & \multirow[t]{2}{*}{ Continuous ECG monitoring } & HR: 50 to 100 & \multirow{2}{*}{$\begin{array}{l}\text { Continuous ECG when the patient is } \\
\text { supine or sitting in chair. Discontinued } \\
\text { during mobilisation. Diagnostic ECG on } \\
\text { indication. If arrhythmia or ischaemia is } \\
\text { detected the treatment goals are adjusted } \\
\text { to current recommendations }\end{array}$} \\
\hline & & No ischemia & \\
\hline Diuresis & Every hour & $\geq 0.5 \mathrm{~mL} / \mathrm{kg} / \mathrm{h}$ & $\begin{array}{l}\text { During mobilisation the diuresis is } \\
\text { summed every third hour }\end{array}$ \\
\hline Temperature & Every $8 \mathrm{~h}$ & $36^{\circ} \mathrm{C}$ to $38^{\circ} \mathrm{C}$ & \\
\hline \multirow[t]{2}{*}{ Pain Visual Assessment Score } & \multirow[t]{2}{*}{ Every $8 \mathrm{~h}$} & VAS: 0 to 2 during rest & \multirow[t]{2}{*}{ No VAS scoring during sleep } \\
\hline & & $\overline{\text { Epidural: Able to move both legs }}$ & \\
\hline Central venous pressure & Every $8 \mathrm{~h}$ & 8 to $12 \mathrm{mmHg}$ & $\begin{array}{l}\mathrm{CVP} \text { and } \mathrm{S}_{C} \mathrm{VO}_{2} \text { is only registered if there } \\
\text { is a central venous catheter in place. The } \\
\text { central venous catheter is removed when } \\
\text { possible }\end{array}$ \\
\hline
\end{tabular}

\begin{tabular}{|c|c|c|c|}
\hline Central venous oxygen saturation & Every $8 \mathrm{~h}$ & $\mathrm{SpO}_{2} \geq 70 \%$ & \\
\hline Standard blood samples & Every $24 \mathrm{~h}$ & Within normal reference values & $\mathrm{Hgb} \geq 4.5 \mathrm{mmol} / \mathrm{L}$ \\
\hline
\end{tabular}

\begin{tabular}{llll}
\hline Treatment (if needed) & Maximal treatment level & Treatments goals & Comments \\
\hline Single sympathomimetic drug support & Continuously & MAP: $\geq 65 \mathrm{mmHg}$ & \\
\cline { 3 - 4 } & & Diuresis: $\geq 0.5 \mathrm{~mL} / \mathrm{kg} / \mathrm{h}$ & Unless contraindicated, oxygen therapy is
\end{tabular}
discontinued when oxygenation is $\geq 94 \%$ without oxygen therapy. During nights: minimum $2 \mathrm{~L}$ supplemental oxygen is given

Positive Expiratory Pressure (PEP) therapy Assistance to PEP therapy: $\quad \mathrm{SpO}_{2} \geq 94 \% \quad$ If the patient does not need assistance once per hour with PEP therapy, guidance in selfadministration of PEP therapy must be available

\begin{tabular}{|c|c|c|c|}
\hline Non-invasive ventilation & Continuously & Normocapnia and norn & \\
\hline \multirow[t]{4}{*}{ Volume / Fluid therapy } & \multirow[t]{4}{*}{ Continuously } & MAP: $\geq 65 \mathrm{mmHg}$ & \multirow{4}{*}{$\begin{array}{l}\text { Fluid balance: Evaluation frequency in } \\
\text { accordance with monitoring level and } \\
\text { vital signs }\end{array}$} \\
\hline & & Diuresis: $\geq 0.5 \mathrm{~mL} / \mathrm{kg} / \mathrm{h}$ & \\
\hline & & $\mathrm{S}_{\mathrm{c}} \mathrm{VO}_{2} \geq 70 \%$ & \\
\hline & & CVP: 8 to $12 \mathrm{mmHg}$ & \\
\hline
\end{tabular}

During evening and night shifts: Staff specialist in anaesthetist/intensive care medicine on in-house duty and staff specialist in surgery on call. ${ }^{a}$ The minimal monitoring level is exceeded when necessary (for example, deterioration).

${ }^{\mathrm{b}}$ All treatment goals are adjusted to the individual patient's co-morbidities, physiological status and in the event of complications in agreement with current recommendations (for example, troponin $\mathrm{T} / \mathrm{I}$ is measured when cardiac ischaemia is suspected).

night, re-evaluation is made. If patients are stable, and local discharge criteria are met, they can be transferred to the surgical ward.

\section{Ward care}

Patients allocated to ward care (WC) are transferred to the surgical ward with a protocol-based discharge note using a standard form (Additional file 5: Anaesthetist - discharge note). Patients are thoroughly evaluated before transfer, and a written plan of treatment and monitoring for the first $24 \mathrm{~h}$ in the ward is outlined in the medical chart. Otherwise, surgical ward treatment is as standard for the individual ward. In general, the participating surgical wards have the resources and facilities to meet the 
Table 2 Surgical ward care: an overview of facilities

\begin{tabular}{|c|c|c|c|}
\hline Observation & Monitoring level & Treatments goals $^{\mathrm{a}}$ & Comments \\
\hline Level of consciousness & Every $8 \mathrm{~h}$ & GCS: 15 & \\
\hline Respiratory rate & Every $8 \mathrm{~h}$ & RR: 10 to 20 & \\
\hline Oxygenation & Every $8 \mathrm{~h}$ & $\mathrm{SpO}_{2} \geq 94 \%$ & Continuous pulse oximetry is not available \\
\hline Blood pressure & Every $8 \mathrm{~h}$ & MAP: 65 to $110 \mathrm{mmHg}$ & \\
\hline \multirow[t]{2}{*}{ Heart rate } & \multirow[t]{2}{*}{ Every $8 \mathrm{~h}$} & HR: 50 to 100 & \multirow{2}{*}{$\begin{array}{l}\text { Continuous ECG is not available. Diagnostic } \\
\text { ECG on indication. If arrhythmia or ischaemi } \\
\text { is detected the treatment goals are adjusted } \\
\text { to current recommendations }\end{array}$} \\
\hline & & No ischaemia & \\
\hline Diuresis & Every $8 \mathrm{~h}$ & $\geq 0.5 \mathrm{~mL} / \mathrm{kg} / \mathrm{h}$ & \\
\hline Temperature & Every $8 \mathrm{~h}$ & $36^{\circ} \mathrm{C}$ to $38^{\circ} \mathrm{C}$ & \\
\hline Pain Visual Assessment Score & Every $8 \mathrm{~h}$ & $\begin{array}{l}\text { VAS: } 0 \text { to } 2 \text { during rest Epidural: } \\
\text { Able to move both legs }\end{array}$ & \\
\hline Central venous pressure & Not available & & \\
\hline Central venous oxygen saturation & Not available & & \\
\hline \multirow[t]{2}{*}{ Standard blood samples } & \multirow[t]{2}{*}{ Every $24 \mathrm{~h}$} & \multirow[t]{2}{*}{ Within normal reference values } & $\mathrm{Hgb} \geq 4.5 \mathrm{mmol} / \mathrm{L}$ \\
\hline & & & $\mathrm{Hgb} \geq 6.0$ during sepsis or heart disease \\
\hline Treatment (if needed) & Maximal treatment level & Treatments goals & Comments \\
\hline Infusion of sympathomimetic drugs & \multicolumn{3}{|l|}{ Not available } \\
\hline Oxygen therapy on open air systems & Continuously & $\mathrm{SpO}_{2} \geq 94 \%$ & $\begin{array}{l}\text { Unless contraindicated. Oxygen therapy is } \\
\text { discontinued when oxygenation is above } \\
\geq 94 \% \text { without oxygen therapy. During } \\
\text { nights: minimum } 2 \text { L supplemental oxygen } \\
\text { is given }\end{array}$ \\
\hline Positive Expiratory Pressure (PEP) therapy & $\begin{array}{l}\text { Assistance to PEP therapy: } \\
\text { every fourth hour during } \\
\text { day and evening shift }\end{array}$ & $\mathrm{SpO}_{2} \geq 94 \%$ & $\begin{array}{l}\text { If the patient does not need assistance with } \\
\text { PEP therapy, guidance in self-administration } \\
\text { of PEP therapy is available }\end{array}$ \\
\hline Non-invasive ventilation & Not available & & \\
\hline \multirow[t]{2}{*}{ Volume / Fluid therapy } & \multirow[t]{2}{*}{ Continuously } & $\begin{array}{l}\text { Systolic blood pressure: } \\
\geq 100 \mathrm{mmHg}\end{array}$ & \multirow{2}{*}{$\begin{array}{l}\text { Fluid balance: Evaluation frequency in } \\
\text { accordance with monitoring level and vital } \\
\text { signs }\end{array}$} \\
\hline & & Diuresis: $\geq 12 \mathrm{~mL} / \mathrm{kg} /$ day & \\
\hline
\end{tabular}

During evening and night shifts: Resident in surgery on in-house duty and staff specialist in surgery on call. Staff specialist in anaesthesiology/intensive care medicine on call from in-house duty.

${ }^{a}$ All treatment goals are adjusted to the individual patient's co-morbidities, physiological status and in the event of complications in agreement with current recommendations.

monitoring and treatment levels outlined in Table 2. This level can only be exceeded for a short time, and it is not possible to initiate continuous monitoring of vital signs. If patients deteriorate they are transferred to an intermediate care, or intensive care unit to be treated appropriately.

\section{Baseline data}

After inclusion we register the following demographic characteristics: dementia; previous stroke; chronic obstructive pulmonary disease; previous pulmonary embolism; previous myocardial infarction; chronic kidney dialysis; cancer; tobacco habit; alcohol consumption; assistance with personal hygiene; The American Society of Anaesthesiologists' Physical Status Classification (ASA) score, and the postoperative sepsis score: grade 0 , no sepsis and no systemic-inflammatory-response-syndrome (SIRS); grade 1, SIRS; grade 2, sepsis; grade 3, severe sepsis; and grade 4, septic shock [35]. We collect the following perioperative data: nature and duration of surgery; method of anaesthesia; location and duration of post-anaesthesia care; infusion of sympathomimetic drug (yes/no); blood loss; volume of crystalloid and/or colloid infusion; and infusion of blood products (type and number of units).

\section{Intervention period data}

We measure compliance with the trial protocol by registering the frequency of observations of the measures listed in Table 3, made during the $48 \mathrm{~h}$ intervention period. Additionally, we register the following measures regarding level of treatment and monitoring in both groups: early-warning-score system [36] monitoring for $>24 \mathrm{~h}$ (yes/no); nutrition initiated within $24 \mathrm{~h}$ (yes/no); mobilisation within $24 \mathrm{~h}$ of surgery (yes/no; level); mobilisation within $48 \mathrm{~h}$ of surgery (yes/no; level); intensivist or anaesthetist evaluation (number; specialist/ non-specialist), and surgeon evaluation (number; specialist/non-specialist). Additionally, we register the use of diagnostic imaging: chest X-ray; lung scintigraphy; and 
Table 3 Compliance with trial protocol

\begin{tabular}{|c|c|c|}
\hline Measures & IC Group (day 2) & WC Group (day 14) \\
\hline \multicolumn{3}{|l|}{ Actual monitoring level } \\
\hline Level of consciousness (number of registrations) & $x$ & $x$ \\
\hline Respiratory rate (number of registrations) & $x$ & $x$ \\
\hline Continuous pulse oximetry (yes/no) & $x$ & $x$ \\
\hline Blood pressure (number of registrations) & $x$ & $x$ \\
\hline Continuous ECG monitoring (yes/no) & $x$ & $x$ \\
\hline 24-h diuresis (number of registrations) & $x$ & $x$ \\
\hline Hourly diuresis registration for $>24$ h (yes/no) & $x$ & $x$ \\
\hline Temperature (number of registrations) & $x$ & $x$ \\
\hline Pain Visual Assessment Score (no. of registrations) & $x$ & $x$ \\
\hline Central venous pressure (number of registrations) & $x$ & $x$ \\
\hline Central venous oxygen saturation (number of samples) & $x$ & $x$ \\
\hline Standard blood samples (number of samples) & $x$ & $x$ \\
\hline \multicolumn{3}{|l|}{ Treatment level } \\
\hline Infusion of sympathomimetic drugs (yes/no) & $x$ & $x$ \\
\hline Parallel infusion of sympathomimetic drugs (yes/no) & $x$ & $x$ \\
\hline$>2$ L supplemental oxygen during nights (yes/no) & $x$ & $x$ \\
\hline Assistance to PEP therapy (number of treatments) & $x$ & $x$ \\
\hline Non-invasive ventilation (yes/no) & $x$ & $x$ \\
\hline Invasive ventilation (yes/no) & $x$ & $x$ \\
\hline Emergency dialysis (yes/no) & $x$ & $x$ \\
\hline 24-h fluid balance calculation (number of registrations) & $x$ & $x$ \\
\hline Protocol-based discharge by anaesthetist (yes/no) & & $x$ \\
\hline Protocol-based round by intensivist (number) & $x$ & \\
\hline Protocol-based round by surgeon (number) & $x$ & \\
\hline Patient location (hours) ${ }^{c}$ & $x$ & $x$ \\
\hline
\end{tabular}

Data stem from medical charts, nurse charts and observation charts used in the 48-h intervention period.

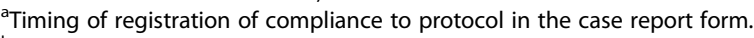

${ }^{b}$ Registered by the anaesthetist writing the discharge note and checked at day 14.

'Post-anaesthesia care unit; intermediate care bed; intensive care bed; surgical ward; medical ward; and/or coronary care unit.

echocardiography. In the IC group we register reason for: premature or late transfer to the surgical ward (respectively before or after $48 \mathrm{~h}$ intervention); 'step-up' to an intensive care bed; the Simplified Acute Physiology Score (SAPS) II [37]; the Sequential Organ Failure Assessment (SOFA) score (day 1 and 2) [38]; and the sepsis score (day 2).

\section{Follow-up}

Mortality data will be retrieved from The Danish Civil Registration System (CRS) [39]. The CRS contains mortality data on all Danish citizens, through a unique personal identification number. The retrieval from CRS will be made by an independent data manager at Copenhagen Trial Unit at interim analysis and at day 30 after the last patient has been enrolled. The secondary outcome measures are registered from the medical chart at day 30 postoperatively, and from the National Patient Registry (NPR), which contains data on hospitalisation of all Danish citizens [40]. Furthermore, return to operating theatre, and postoperative medical and surgical complications requiring treatment within 14 days of randomisation, are retrieved from the medical chart at day 30 .

\section{Outcome measures}

The primary outcome measure is all-cause 30-day mortality.

Secondary outcome measures are: (1) all-cause mortality within the total observation time, measured 30 days after the last included patient; (2) admission to an intensive care unit, and duration thereof, within 30 days of randomisation; and (3) duration of hospitalisation postoperatively (days).

\section{Data collection and trial conduct}

Data are collected on printed case report forms, on which a unique barcode number is printed to eliminate the possibility of duplication of the case report forms. 
Case report forms are scanned into the database using the Verity Teleform ${ }^{\circledR}$ system (Verity, Sunnyvale, CA, USA) managed by Copenhagen Trial unit. Mortality data is extracted from The CRS. The Steering Committee will not have access to the dataset until the trial has been completed. The InCare trial is conducted in compliance with the Helsinki Declaration, and is approved by the Copenhagen Capital Region Ethical board (H-3-2010010) and the Danish Data Protection Agency (HEH.afd. I.750.16-18). The trial is registered at clinicaltrials.gov (NCT01209663), and conducted and monitored in accordance with the ICH-GCP guidelines. Case report forms are checked for validity and internal consistency through trial site visits by Good Clinical Practice (GCP) monitors, who checks source data in approximately $10 \%$ of the case report forms selected at random. The trial is designed in compliance with the Consort Statement, and the two extensions: 'Improving reporting of pragmatic trials' and 'Extending the consort statement to randomised trials of nonpharmacologic trials' [41-43].

\section{Interim analyses}

We have established an independent Data Monitoring Committee (DMC) to evaluate safety and efficacy on scheduled interim analyses of 30-day mortality data. The monitoring plan is based on the modified Haybittle-Peto boundaries for stopping trials after interim analyses in the second half of the inclusion period $[44,45]$. The mortality data are presented to the DMC under a blinded code for allocation group. The first interim analysis will be conducted when 30-day mortality data of 200 trial participants have been obtained, and/or 75 deaths have been documented during the trial. If the first interim analysis of 30-day mortality data is significant $(P<0.001)$ for benefit or harm from the intervention, a second interim analysis will be made when 30-day mortality data of 300 trial participants have been obtained and/or an additional 25 deaths have been documented. If the second interim analysis is also statistically significant $(P<0.001)$ for benefit or harm from the intervention the DMC can advise the steering committee to stop the trial.

\section{Statistical analysis}

The outcome measures will be analysed for all randomised patients in a modified intention-to-treat analysis leading to the primary results of the trial [46]. Patients with the following major protocol violations will not be included in a per-protocol analysis: not fulfilling an inclusion criterion; fulfilling an exclusion criterion; IC for $<48 \mathrm{~h}$ because of early discharge to the ward. The primary outcome measure, 30-day mortality, will be analysed with an unadjusted univariate logistic regression, and a multivariate logistic regression analysis will be made adjusting for stratification variables: trial-site, APACHE II score (10 to 14 or $\geq 15$ ), and perforated viscera (yes versus no); and design variables: age, ASA score ( 1 to 2 or $\geq 3$ ), cancer, and nature of surgery (+/- re-operation). The secondary outcome measure, survival within the total observation time, will be analysed with an unadjusted Cox regression analysis, and a Cox regression analysis adjusted for the above-mentioned stratification variables and design variables [47]. Survival within the total observation time will be illustrated with Kaplan-Meier estimates. Fisher's exact or chi-squared tests will be used, depending on values, to analyse the differences of frequencies of registered complications in the two groups. Mortality data will be retrieved on patients registered as 'not included in the trial due to lack of IC bed'. The data on these patients will be compared with that from the WC group, then included with the WC group data and compared with the IC group. Data on a semi-quantitative scale will be analysed using the Mann-Whitney test. Double sided $P$ values $<0.05$ will be considered statistically significant.

\section{Sample size}

Emergency major abdominal surgical patients with an APACHE II score $\geq 10 / 11$ have a $28 \%$ to $45 \%$ mortality rate [27-31]. We assume that patients with an APACHE II score of $\geq 10$ have a 30 -day mortality of $38 \%$. We aim to confirm or reject an intervention effect of $13 \%$ absolute risk reduction in mortality (a relative risk reduction of $34 \%$ ), in accordance with the difference between group mortality detected in our recent observational study of optimised perioperative care in perforated peptic ulcer patients [48]. The sample size with a type 1 error risk of $5 \%$, and a type 2 error risk of $20 \%$ ( $80 \%$ power), was estimated to 200 patients in each group [49].

\section{Ethical considerations}

Randomised trials in presumed optimised health-care protocols are associated with unique ethical problems, especially when mortality is the outcome measure. Patients often intuitively find the IC appealing when the interventions are outlined for them, but IC is only available by chance if they consent to participate in the trial. In this design it is of utmost importance to consider that the effect of postoperative intermediate care, with a package of possible interventions, remains to be proven. Furthermore, participants are exclusively enrolled postoperatively when they fulfill the current discharge criteria to be transferred to the surgical ward, which ensures that the control group is given the best current available standard treatment.

In the InCare trial, all diagnostic tests and interventions in the control and intervention group are initiated by the attending doctors, thereby reflecting their medical judgment, and not alone the trial protocol. We are thus only evaluating the effect of the extra monitoring, extra 
specialist attention and patient placement in IC or WC with differences in routine, resources, and capability.

\section{Trial management}

The InCare trial is investigator initiated, controlled by a steering committee including anaesthetists, intensivists and surgeons, all with solid research experience. The committee is responsible for the planning, design and conduct of the trial. At each trial site two or three site investigators with at least one surgeon and one intensivist/anaesthetist manage the local implementation. This includes teaching and supervising of staff, daily management, and data collection with support from the principal coordinating investigator. Protocol observance is stimulated by repeated staff educational sessions on patient enrolment, and execution of the trial interventions by the site investigators and the coordinating investigator. Additionally, intervention period data are checked to monitor adherence to protocol, thereby giving feedback to staff. A monthly newsletter with trial updates is sent to the involved parties, and published on the trial website.

\section{Discussion}

Trial rationale

Participants

Our recent findings in emergency surgical patients with perforated ulcer [48] suggested that we should investigate the effects of IC in emergency major abdominal surgical patients with an APACHE II score $\geq 10$. The APACHE II index is well-known to anaesthetists responsible for patient enrolment, and it reflects the presence of perioperative sepsis, cardiovascular and respiratory failure.

\section{Interventions}

The level of care in the IC group is based on national and international definitions, descriptions, surveys and recommendations which may increase the external validity [15,50-53].

We chose an IC admission duration of $48 \mathrm{~h}$ influenced by the findings of Garmil et al. [54] that $80 \%$ of patients dying or disabled in hospital began to deteriorate within 24 h postoperatively, and of Jhanji et al. [13] that surgical ward admission was approximately $48 \mathrm{~h}$ prior to the necessary postoperative intensive care unit admission. Furthermore, this duration of the IC intervention was conceived realistic in that it could be accepted by the participating trial sites.

Daily protocol-based rounds are made in the IC group to ensure uniform evaluation at the participating trial sites, thus improving internal validity. The rounds made by surgeons and intensivists, are aimed at optimising pain relief, cardiopulmonary function, fluid balance, nutrition, mobilisation and early detection of complications.
The WC group receives ward care according to local standards. We choose not to reinforce a treatment and monitoring protocol in the WC group, as we wish to compare IC with standard care at the participating trial sites. With trial site stratified randomisation, we aim to adjust for possible differences in standard care at the wards. Additionally, we register intervention period data on level of treatment and monitoring as in the IC group, which enable us to report the actual level of treatment and monitoring in the wards.

In the WC group, intervention period data collection is made on day 14, because data collection on day 2 would equate with an extra clinical evaluation and the possible initiation of treatment, which could differ from current standard care.

\section{Previous research}

The effect of postoperative IC is unresolved in highevidence studies. Previous studies of IC have been nonrandomised, observational or descriptive, often with contradictory results [17-23]. Davies et al. and Bellomo et al. $[18,19]$ found no reduction in postoperative inhospital mortality or morbidity following introduction of an IC unit at their facilities. Both studies reported a change in surgical intake tending more to emergency surgery after introduction of the IC unit. This may explain the lack of effect in their unadjusted analyses. Turner et al. [20] found an excess crude mortality rate in patients not admitted to IC units despite requests from the attending surgeon or anaesthetist. In a similar design Mcllroy et al. [21] concluded that IC may lead to longer hospital stay, but no effect on 30-day mortality or all-cause morbidity could be shown. Jones et al. [22] compared two hospitals (one with, and one without, an IC unit). This study suggested that postoperative IC might reduce cardiopulmonary complications after major abdominal surgery, when risk adjusting with Physiologic and Operative Severity Score for the enumeration of mortality and morbidity (POSSUM score). No effect on mortality or length of hospital stay could be shown. Swart et al. [23] compared postoperative intensive care with surgical ward care in colorectal surgical patients with a preoperative anaerobic threshold $<11 \mathrm{~mL}$ oxygen $/ \mathrm{kg} / \mathrm{min}$. They found that there was significant decrease in cardiac adverse events in the intensive care patients, but no effect on in-hospital mortality, or length of hospital stay could be shown.

Common in the previous research is a high risk of bias as confounding by indication and selection bias, and thereby unbalanced number of risk patients in the compared groups is imminent. Furthermore, the cited studies are characterised by limited power to show effect on mortality, because they predominantly investigate patients undergoing scheduled surgical procedures 
which have much lower mortality than emergency abdominal surgical procedures.

\section{Strengths}

The InCare trial is the first randomised clinical trial to evaluate the effect of postoperative intermediate care. The design will provide evidence with low risk of bias compared with previous studies.

We have thoroughly described the complex intervention in the IC group and the compliance with protocol is rigorously registered to improve internal validity, and transparent reporting of the trial. IC is evaluated in high-risk emergency abdominal surgical patients with high mortality. This enables us to report all-cause mortality, in a relevant patient population, which we believe is the most relevant primary outcome measure when evaluating IC. We have chosen not to report postoperative complications as an outcome measure. Our hypothesis is that the IC will lead to avoidance or earlier recognition of postoperative complications. Reporting complications when evaluating IC compared with WC can be misleading in that IC may reduce complications because of early postoperative optimisation, or show an increase in registered complications because of more intensive controls, for example, X-rays/ atelectasis, which might not be detected on the ward and spontaneously resolve. Given that the IC both can lead to a decrease (avoidance of complications) or an increase (recognition of complications) in the registered postoperative complication rate, appropriate conclusions on these outcomes will be difficult in the case of no mortality effect because of type II error. Additionally, if complications were to be reported as an outcome measure, a detailed prospective bedside registration during the first 14 to 30 days after surgery would be necessary. These investigator follow-up visits would be equivalent to extra clinical evaluations of the patients, which might well entail change in the current standard care in the IC and WC groups. This could potentially jeopardise the external validity of the primary outcome measure and conceal a beneficial effect of IC on mortality.

\section{Limitations}

In this pragmatic clinical trial some important limitations must be noted. Unfortunately, it is not possible to blind the involved healthcare personnel, or the participants, in this trial, and outcome assessment is only partially blinded. Given that the data on mortality are retrieved from The Danish Civil Registration System the risk of detection bias is minimal compared to, for example, an assessment of postoperative complications as an outcome. The lack of blinding, and the fact that the same surgeons at the same hospital treat both the IC and WC group may insinuate a learning bias possibly concealing a beneficial effect of the IC. In the InCare trial the WC group receives local standard care, which is not rigorously defined by the protocol. This is a limitation. Our efforts to register intervention period data will provide important information on the actual monitoring and treatment given to the WC group, and will also expose any learning bias in the WC group.

The intervention is complex, and the involvement of several trial sites entails the risk that not all elements of intervention are uniformly applied. Nevertheless, these deviations may reflect clinical practice, and the transparent reporting will give ground for a fair interpretation of the trial result. Finally, as this trial is the first of its kind, the sample size calculation is based on estimates, which entails a risk of type II error, thus not detecting a smaller intervention effects than the anticipated relative risk reduction of $34 \%$ used in our sample size estimation.

\section{Conclusion}

The InCare trial is the first randomised clinical trial that aims to contribute to answer the important research question: should high-risk surgical patients receive postoperative intermediate care. The results may potentially influence the survival of many high-risk surgical patients. As a pioneer trial in the area, it will provide important data on the feasibility of a future large randomised clinical trial, which may definitively settle the question.

\section{Trial status}

All Danish public-healthcare centres that receive emergency major abdominal surgical patients were asked to participate in the trial $(n=23)$. The reasons for declining to participate were: limited intensive/intermediate care facilities $(n=8)$; high-risk surgical patients treated $>24 \mathrm{~h}$ postoperatively in a post-anaesthesia care unit or an intermediate/intensive care unit $(n=3)$; conflicting research projects $(n=2)$; lack of resources to conduct more than one multicentre clinical trial $(n=1)$, and no answer after multiple contacts $(n=2)$.

Seven centres thus currently participate in the trial and have started enrolment: Herlev Hospital, 4 October $2010(n=80)$; Koege Hospital, 11 October $2010(n=35)$; Hilleroed Hospital, 15 November $2010(n=36)$; Herning Hospital, 2 May $2011(n=19)$; Aabenraa Hospital, 1 July $2011(n=2)$; Bispebjerg Hospital, 1 December 2011 $(n=17)$; and Vejle Hospital, 1 February $2012(n=10)$. To date (1 June 2012) 199 patients have been enrolled.

\section{Additional files}

Additional file 1: Search string.

Additional file 2: National discharge recommendations.

Additional file 3: Surgeon - protocol-based round.

Additional file 4: Intensivist - protocol-based round.

Additional file 5: Anaesthetist - discharge note. 


\section{Abbreviations}

APACHE: Acute Physiology and Chronic Health Evaluation; ASA: American Society of Anaesthesiologists physical status classification score; BP: Blood pressure; CENTRAL: Cochran Central Register of Controlled Trials; CPAP: Continuous positive airway pressure; CRP: C-reactive protein; CRS: The Civil Registration System; CTU: Copenhagen Trial Unit; CVP: Central venous pressure; DMC: Data monitoring committee; DVT: Deep venous thrombosis; ECG: Electrocardiogram; GCS: Glasgow Coma Scale; HGB: Haemoglobin; HR: Heart rate; IC: Intermediate care; ICH-GCP: International Conference on Harmonization Good Clinical Practice; MAP: Mean arterial pressure; NPR: National Patient Registry; PEP: Positive expiratory pressure; POSSUM: Physiologic and Operative Severity score for the enumeration of mortality and morbidity; RBC: Red blood count; RR: Respiratory rate; SAPS II: Simplified Acute Physiology Score II; $\mathrm{S}_{\mathrm{CO}_{2}}$ : Central venous saturation; SIRS: Systemic-Inflammatory-Response-Syndrome; SOFA: Sequential Organ Failure Assessment score; $\mathrm{SpO}_{2}$ : Oxygen saturation; TP: Temperature; VAS: Pain Visual Assessment Score; WC: Ward care; WBC: White blood count

\section{Competing interests}

The authors declare that they have no competing interests.

\section{Authors' contributions}

Each author has made substantial intellectual contributions to the conception, design or implementation of The InCare trial and has been involved in the critical revision of the manuscript. All authors have approved the final manuscript for submission. MVA is the principal coordinating investigator. MVA, TW, JW, MHM, JR, LNJ and AMM are members of the steering committee. TW, IG, HLJ, EGH, LMP, JS, EKS, MB, JV, IR, REB, JP, MRM, $T F, M J B, J A, C M, M R, C J, L B$ and $H \varnothing$ are principal site investigators and have coordinated the implementation, enrolment, intervention or follow-up at their centre. AMM is the sponsor.

\section{Acknowledgements}

The authors wish to thank the following, who also helped as, site investigators but do not fulfil authorship criteria: Per Bo Larsen (Herlev Hospital), Henrik Jørgensen (Herlev Hospital), Jon Jacobsen (Hillerød Hospital), Lene Höegh (Hillerød Hospital), Michael Lindhardt Rasmussen (Herning Hospital), Anne-Marie Ulrik (Aabenraa Hospital), Lise Kaag (Aabenraa Hospital) and Kirsten Møller (Bispebjerg Hospital). We thank the participants, staff (anaesthetists, intensivists, surgeons, nurses and secretaries) and the head of departments on the participating sites who have made this trial possible. Furthermore, we wish to thank Torsten Faber for programming the APACHE II calculator and John Neville Mitchell for copy-editing. The trial is funded by The Department of Anaesthesiology and Intensive Care Medicine at Herlev University Hospital, The Research Council of Herlev University Hospital, The Danish Capital region's Medical Research Foundation, The Health Insurance Foundation (2010B053), A.P. Møller Foundation for the Advancement of Medical Science, The Danish Council for Independent Research in Medical Science (11-108561), The Research Council of Hospital of Southern Jutland Aabenraa, and The Lundbeck Foundation (R118-A11357). None of the funding sources have influence on the trial design, data collection, data management, data analysis or reporting.

\section{Data Monitoring Committee}

Lars Simon Rasmussen MD, DMSc. Department of Anaesthesiology, Centre of Head and Orthopaedics, Rigshospitalet, Copenhagen University Hospital, Copenhagen Denmark and Ismail Gögenur, MD, DMSc. Department of Surgery, Herlev Hospital, Copenhagen University, Herlev, Denmark.

\section{Quality assessment and Good Clinical Practice monitoring}

Thordis Thomsen, RN, PhD. Department of Anaesthesiology, Abdominal Centre, Rigshospitalet, Copenhagen University Hospital, Copenhagen, Denmark and Ulla Tølbø\|ll Jensen, laboratory technician, Research Unit, Department of Anaesthesiology and Intensive Care Medicine, Herlev Hospital, Copenhagen University, Herlev, Denmark.

\section{Randomisation and data management}

Copenhagen Trial Unit, Centre for Clinical Intervention Research, Rigshospitalet, Copenhagen University Hospital, Copenhagen, Denmark.

\section{Author details}

${ }^{1}$ Department of Anaesthesiology and Intensive Care Medicine, Herlev Hospital, Copenhagen University, Herlev Ringvej 75, DK-2730 Herlev, Denmark. ${ }^{2}$ Copenhagen Trial Unit, Centre for Clinical Intervention Research, Rigshospitalet, Copenhagen University Hospital, Copenhagen, Denmark. ${ }^{3}$ Department of Anaesthesiology and Intensive Care Medicine, Copenhagen University Hospital Rigshospitalet, Copenhagen, Denmark. ${ }^{4}$ Department of Surgery, Herlev Hospital, Copenhagen University, Herlev, Denmark. ${ }^{5}$ Department of Surgery, Bispebjerg Hospital, Copenhagen University, Copenhagen, Denmark. ${ }^{6}$ Department of Anaesthesiology, Koege Hospital, Copenhagen University, Koege, Denmark. ${ }^{7}$ Department of Surgery, Koege Hospital, Copenhagen University, Koege, Denmark. ${ }^{8}$ Department of Anaesthesiology, Hilleroed Hospital, Copenhagen University, Hilleroed, Denmark. ${ }^{9}$ Department of Surgery, Hilleroed Hospital, Copenhagen University, Hilleroed, Denmark. ${ }^{10}$ Department of Anaesthesiology, Herning Regional Hospital, Herning, Denmark. ${ }^{11}$ Department of Surgery, Herning Regional Hospital, Herning, Denmark. ${ }^{12}$ Department of Anaesthesiology, Hospital of Southern Jutland Aabenraa, Aabenraa, Denmark. ${ }^{13}$ Department of Surgery, Hospital of Southern Jutland Aabenraa, Aabenraa, Denmark.

${ }^{14}$ Department of Anaesthesiology, Bispebjerg Hospital, Copenhagen University, Copenhagen, Denmark. ${ }^{15}$ Department of Anaesthesiology, Hospital Lillebaelt Vejle, Vejle, Denmark. ${ }^{16}$ Department of Surgery, Hospital Lillebaelt Vejle, Vejle, Denmark.

Received: 15 June 2012 Accepted: 25 January 2013

Published: 2 February 2013

\section{References}

1. Weiser TG, Regenbogen SE, Thompson KD, Haynes AB, Lipsitz SR, Berry WR, Gawande AA: An estimation of the global volume of surgery: a modelling strategy based on available data. Lancet 2008, 372:139-144.

2. Glance LG, Lustik SJ, Hannan EL, Osler TM, Mukamel DB, Qian F, Dick AW: The surgical mortality probability model: derivation and validation of a simple risk prediction rule for noncardiac surgery. Ann Surg 2012, 255:696-702.

3. Pedersen T, Eliasen $\mathrm{K}$, Henriksen E: A prospective study of mortality associated with anaesthesia and surgery: risk indicators of mortality in hospital. Acta Anaesthesiol Scand 1990, 34:176-182.

4. Pearse RM, Harrison DA, James P, Watson D, Hinds C, Rhodes A, Grounds RM, Bennett ED: Identification and characterisation of the high-risk surgical population in the United Kingdom. Crit Care 2006, 10:R81.

5. Garcea G, Ganga R, Neal CP, Ong SL, Dennison AR, Berry DP: Preoperative early warning scores can predict in-hospital mortality and critical care admission following emergency surgery. J Surg Res 2010, 159:729-734.

6. Clarke A, Murdoch H, Thomas MJ, Cook TM, Peden CJ: Mortality and postoperative care after emergency laparotomy. Eur J Anaesthesiol 2011, 28:16-19.

7. Sorensen LT, Malaki A, Wille-Jorgensen P, Kallehave F, Kjaergaard J, Hemmingsen $U$, Moller $L N$, Jorgensen T: Risk factors for mortality and postoperative complications after gastrointestinal surgery. J Gastrointest Surg 2007, 11:903-910.

8. Iversen LH, Bulow S, Christensen IJ, Laurberg S, Harling H: Postoperative medical complications are the main cause of early death after emergency surgery for colonic cancer. Br J Surg 2008, 95:1012-1019.

9. Harten J, McCreath BJ, McMillan DC, McArdle CS, Kinsella J: The effect of gender on postoperative mortality after emergency abdominal surgery. Gend Med 2005, 2:35-40.

10. Khuri SF, Henderson WG, DePalma RG, Mosca C, Healey NA, Kumbhani DJ: Determinants of long-term survival after major surgery and the adverse effect of postoperative complications. Ann Surg 2005, 242:326-341.

11. Ghaferi AA, Birkmeyer JD, Dimick JB: Variation in hospital mortality associated with inpatient surgery. N Engl J Med 2009, 361:1368-1375.

12. Danish National Board of Health: Evaluation of the Intensive Care Capacity. Copenhagen: Danish National Board of Health; 2004.

13. Jhanji S, Thomas B, Ely A, Watson D, Hinds CJ, Pearse RM: Mortality and utilisation of critical care resources amongst high-risk surgical patients in a large NHS trust. Anaesthesia 2008, 63:695-700.

14. Ridley SA: Intermediate care, possibilities, requirements and solutions. Anaesthesia 1998, 53:654-664.

15. Danish National Board of Health: Intensive Care - Definitions, Documentation and Visitation. Copenhagen: Danish National Board of Health; 2006. 
16. Nasraway SA, Cohen IL, Dennis RC, Howenstein MA, Nikas DK, Warren J, Wedel SK: Guidelines on admission and discharge for adult intermediate care units. American College of Critical Care Medicine of the Society of Critical Care Medicine. Crit Care Med 1998, 26:607-610.

17. Turner J, Gemmell LW: Are high dependency units effective and efficient? A systemic review of the evidence. Clin Intensive Care 2003, 14:123-133.

18. Davies J, Tamhane R, Scholefield C, Curley P: Does the introduction of HDU reduce surgical mortality? Ann $R$ Coll Surg Engl 1999, 81:343-347.

19. Bellomo R, Goldsmith D, Uchino S, Buckmaster J, Hart G, Opdam H, Silvester W, Doolan L, Gutteridge G: A before and after trial of the effect of a highdependency unit on post-operative morbidity and mortality. Crit Care Resusc 2005, 7:16-21.

20. Turner M, McFarlane HJ, Krukowski ZH: Prospective study of high dependency care requirements and provision. J R Coll Surg Edinb 1999, 44:19-23.

21. Mcllroy DR, Coleman BD, Myles PS: Outcomes following a shortage of high dependency unit beds for surgical patients. Anaesth Intensive Care 2006, 34:457-463.

22. Jones HJ, Coggins R, Lafuente J, De CL: Value of a surgical highdependency unit. Br J Surg 1999, 86:1578-1582.

23. Swart $M$, Carlisle JB: Case-controlled study of critical care or surgical ward care after elective open colorectal surgery. Br J Surg 2012, 99:295-299.

24. Knaus WA, Draper EA, Wagner DP, Zimmerman JE: APACHE II: a severity of disease classification system. Crit Care Med 1985, 13:818-829.

25. Ohmann C, Wittmann DH, Wacha H: Prospective evaluation of prognostic scoring systems in peritonitis. Peritonitis Study Group. Eur J Surg 1993, 159:267-274.

26. Alvarez JA, Baldonedo RF, Bear IG, Truan N, Pire G, Alvarez P: Presentation, treatment, and multivariate analysis of risk factors for obstructive and perforative colorectal carcinoma. Am J Surg 2005, 190:376-382.

27. Wacha H, Hau T, Dittmer R, Ohmann C: Risk factors associated with intraabdominal infections: a prospective multicenter study. Peritonitis Study Group. Langenbecks Arch Surg 1999, 384:24-32.

28. Pacelli F, Doglietto GB, Alfieri S, Piccioni E, Sgadari A, Gui D, Crucitti F: Prognosis in intra-abdominal infections. Multivariate analysis on 604 patients. Arch Surg 1996, 131:641-645.

29. Kulkarni SV, Naik AS, Subramanian N Jr: APACHE-Il scoring system in perforative peritonitis. Am J Surg 2007, 194:549-552.

30. Schein M, Gecelter G, Freinkel Z, Gerding H: APACHE II in emergency operations for perforated ulcers. Am J Surg 1990, 159:309-313.

31. Setti Carraro PG, Magenta A, Segala M, Ravizzini C, Nespoli A, Tiberio G: Predictive value of a pathophysiological score in the surgical treatment of perforated diverticular disease. Chir Ital 1999, 51:31-36.

32. Wunsch $H$, Angus DC, Harrison DA, Collange O, Fowler R, Hoste EA, de Keizer NF, Kersten A, Linde-Zwirble WT, Sandiumenge A, Rowan KM: Variation in critical care services across North America and Western Europe. Crit Care Med 2008, 36:2787-2789.

33. Danish Society of Anaesthesiology and Intensive Care Medicine: Recommendations for Choice of Discharge Criteria from Anaesthesiology Department to the Surgical Ward After Anaesthesia. 50th edition. Copenhagen: Danish Society of Anaesthesiology and Intensive Care Medicine; 2004. http://www.dasaim.dk/images/stories/PDF/ Rekommendationer/rekomm_udarb_udskrivkrit_til_kir_stamafd.pdf.

34. InCare trial. 2010, www.incaretrial.dk

35. Dellinger RP, Levy MM, Carlet JM, Bion J, Parker MM, Jaeschke R, et al: Surviving sepsis campaign: international guidelines for management of severe sepsis and septic shock: 2008. Intensive Care Med 2008, 34:17-60.

36. McGaughey J, Alderdice F, Fowler R, Kapila A, Mayhew A, Moutray M: Outreach and Early Warning Systems (EWS) for the prevention of intensive care admission and death of critically ill adult patients on general hospital wards. Cochrane Database Syst Rev 2007, 3:CD005529.

37. Le Gall JR, Lemeshow S, Saulnier F: A new Simplified Acute Physiology Score (SAPS II) based on a European/North American multicenter study. JAMA 1993, 270:2957-2963.

38. Vincent JL, De MA, Cantraine F, Moreno R, Takala J, Suter PM, Sprung CL, Colardyn F, Blecher S: Use of the SOFA score to assess the incidence of organ dysfunction/failure in intensive care units: results of a multicenter, prospective study. Working group on "sepsis-related problems" of the European Society of Intensive Care Medicine. Crit Care Med 1998, 26:1793-1800

39. Pedersen CB: The Danish Civil Registration System. Scand J Public Health 2011, 39:22-25.
40. Lynge E, Sandegaard JL, Rebolj M: The Danish National Patient Register. Scand J Public Health 2011, 39:30-33.

41. Schulz KF, Altman DG, Moher D: CONSORT 2010 statement: updated guidelines for reporting parallel group randomized trials. Ann Intern Med 2010, 152:726-732.

42. Boutron I, Moher D, Altman DG, Schulz KF, Ravaud P: Extending the CONSORT statement to randomized trials of nonpharmacologic treatment: explanation and elaboration. Ann Intern Med 2008, 148:295-309.

43. Zwarenstein M, Treweek S, Gagnier JJ, Altman DG, Tunis S, Haynes B, Oxman AD, Moher D, CONSORT group, Pragmatic Trials in Healthcare (Practihc) group: Improving the reporting of pragmatic trials: an extension of the CONSORT statement. BMJ 2008, 337:a2390.

44. Haybittle JL: Repeated assessment of results in clinical trials of cancer treatment. Br J Radiol 1971, 44:793-797

45. Peto R, Pike MC, Armitage P, Breslow NE, Cox DR, Howard SV, Mantel N, McPherson K, Peto J, Smith PG: Design and analysis of randomized clinical trials requiring prolonged observation of each patient. I. Introduction and design. Br J Cancer 1976, 34:585-612.

46. Fergusson D, Aaron SD, Guyatt G, Hebert P: Post-randomisation exclusions: the intention to treat principle and excluding patients from analysis. $B M$. 2002, 325:652-654.

47. Lewis JA: Statistical principles for clinical trials (ICH E9): an introductory note on an international guideline. Stat Med 1999, 18:1903-1942.

48. Moller MH, Adamsen S, Thomsen RW, Moller AM: Multicentre trial of perioperative protocol to reduce mortality in patients with peptic ulcer perforation. Br J Surg 2011, 98:802-810.

49. PS: Power and Sample Size Calculation. Nashville, TN: Vanderbilt University, Department of Biostatistics; 2009.

50. Crosby DL, Rees GA, Gill J: The role of the high dependency unit in postoperative care: an update. Ann R Coll Surg Engl 1990, 72:309-312.

51. Thompson FJ, Singer M: High dependency units in the UK: variable size, variable character, few in number. Postgrad Med J 1995, 71:217-221.

52. Zimmerman JE, Wagner DP, Sun X, Knaus WA, Draper EA: Planning patient services for intermediate care units: insights based on care for intensive care unit low-risk monitor admissions. Crit Care Med 1996, 24:1626-1632.

53. Danish Society of Intensive Care Medicine: Definition of Intermediate Care Units. Copenhagen: Danish Society of Intensive Care Medicine; 2005.

54. Gamil M, Fanning A: The first $24 \mathrm{~h}$ after surgery. A study of complications after 2153 consecutive operations. Anaesthesia 1991, 46:712-715.

doi:10.1186/1745-6215-14-37

Cite this article as: Vester-Andersen $M$ et al:: Effect of intermediate care on mortality following emergency abdominal surgery. The InCare trial: study protocol, rationale and feasibility of a randomised multicentre trial. Trials 2013 14:37.

\section{Submit your next manuscript to BioMed Central and take full advantage of:}

- Convenient online submission

- Thorough peer review

- No space constraints or color figure charges

- Immediate publication on acceptance

- Inclusion in PubMed, CAS, Scopus and Google Scholar

- Research which is freely available for redistribution 